\title{
Near-infrared study of the stellar population of Sh2-152
}

\author{
S. Ramírez Alegría ${ }^{1,2}$, A. Herrero ${ }^{1,2}$, A. Marín-Franch ${ }^{3,4}$, E. Puga ${ }^{5}$, F. Najarro ${ }^{5}$, \\ J. A. Acosta Pulido ${ }^{1,2}$, S. L. Hidalgo ${ }^{1,2}$, and S. Simón-Díaz ${ }^{1,2}$ \\ ${ }^{1}$ Instituto de Astrofísica de Canarias, 38205 La Laguna, Tenerife, Spain \\ e-mail: [sramirez; ahd; jap; shidalgo;ssimon]@iac.es \\ 2 Departamento de Astrofísica, Universidad de La Laguna, 38205 La Laguna, Tenerife, Spain \\ 3 Centro de Estudios de Física del Cosmos de Aragón (CEFCA), 44001 Teruel, Spain \\ e-mail: amarin@cefca.es \\ 4 Departamento de Astrofísica, Universidad Complutense de Madrid, 38040 Madrid, Spain \\ 5 Centro de Astrobiología (CSIC-INTA), 28850 Torrejón de Ardoz, Madrid, Spain \\ e-mail: [elena;najarro]@damir.iem.csic.es \\ Received 31 December 2010 / Accepted 29 July 2011
}

\section{ABSTRACT}

\begin{abstract}
Context. The discovery of new massive star clusters and massive stellar populations in previously known clusters in our Galaxy by means of infrared studies has changed our view of the Milky Way from an inactive to an active star-forming machine. Within this scenario, we present a near-infrared spectrophotometric study of the stellar content of the compact HII region Sh2-152.

Aims. We aim to determine the distance, extinction, age, and mass of Sh2-152, using for the first time near-infrared stellar classification for several sources in the region.

Methods. Using our near-infrared $\left(J, H\right.$, and $\left.K_{\mathrm{S}}\right)$ photometry and the colour-magnitude diagram for the cluster field, we selected 13 bright stars, candidate members of the reddened cluster's main sequence, for $H$ - and $K$-spectroscopy and spectral classification. This near-infrared information was complemented with an optical spectrum of the ionizing central star to confirm its spectral nature.

Results. From the 13 spectroscopically observed stars, 5 were classified as B-type, 3 as G-type, 2 were young stellar objects (YSOs), and 3 remained unclassified (because of the poor data quality). The cluster's extinction varies from $A_{K_{\mathrm{S}}}=0.5$ to $2.6 \mathrm{mag}$ ( $A_{V}=4.5$ to $24 \mathrm{mag}$ ) and the distance is estimated to be $3.21 \pm 0.21 \mathrm{kpc}$. The age of the cluster is younger than $9.4 \mathrm{Myr}$ and the lower limit to the total mass of the cluster is $(2.45 \pm 0.79) \times 10^{3} M_{\odot}$. We compare the number of ionizing photons emitted from the OB-type stars with the Lyman continuum photons derived from the radio observations and conclude that both quantities are consistent for the central region of Sh2-152. In contrast, the main ionizing source of the lower region remains unidentified.
\end{abstract}

Key words. techniques: spectroscopic - stars: luminosity function, mass function - Hertzsprung-Russell and C-M diagrams - infrared: stars - stars: early-type - open clusters and associations: individual: Sh2-152

\section{Introduction}

Thanks to infrared studies such as DENIS (Epchtein et al. 1997), 2MASS (Skrutskie et al. 2006), GLIMPSE (Benjamin et al. 2003), and UKIDSS (Lawrence et al. 2007), we have been able to observe regions of high visual extinction, previously impossible to achieve with optical instruments, and discover that our Galaxy is an active star-formation machine (Figer 2008), fed by massive clusters and their massive stars.

Some of these massive objects, for example the ArchesQuintuplet cluster (Figer et al. 1999), RSGC1 (Figer et al. 2006; Davies et al. 2008), RSGC2 (Davies et al. 2007), RSGC3 (Clark et al. 2009; Alexander et al. 2009) and Alicante 8 (a.k.a. RSGC4, Negueruela et al. 2010) are newly discovered massive clusters; but others such as CygOB2 (Knödlseder 2000) or Westerlund1 (Clark et al. 2005), are previously known clusters with a newly detected massive stellar population. This increase in massive cluster discoveries has been complemented with new catalogues of cluster candidates, for example Bica et al. (2003a,b), Dutra et al. (2003), Mercer et al. (2005), and Froebrich et al. (2007). If we also consider that hundreds of Galactic massive clusters remain unknown (Hanson \& Popescu 2008), it is easy to grasp the need to generate projects dedicated to finding and characterizing these new massive objects.
This re-evaluation of our ideas of the Milky Way motivated us to develop the MASGOMAS (MAssive Stars in Galactic Obscured MAssive clusterS) project. Within this project, we have completed a photometric near-infrared broad band $(J, H$ and $K_{\mathrm{S}}$ ) catalogue of 44 cluster candidates extracted from previous catalogues (Dutra \& Bica 2001; Bica et al. 2003a,b) and we have continued with a medium resolution multi-object spectroscopic follow-up for 9 of the most promising candidates. A detailed description of MASGOMAS is given in the first paper of the project (Marín-Franch et al. 2009).

One of our candidates is the star-forming compact HII region Sh2-152 $\left(\alpha_{2000}=22^{\mathrm{h}} 58^{\mathrm{m}} 45^{\mathrm{s}}\right.$ and $\left.\delta_{2000}=+58^{\circ} 46^{\prime} 50^{\prime \prime}\right)$. This region is located in the Perseus arm, close to the Galactic plane $(l=108.76, b=-0.95)$ and stands out from the background in the Spitzer $3.6 \mu \mathrm{m}$ image shown in Fig. 1. Sh2-152 contains two IRAS sources: IRAS 22566+5830 and IRAS 22566+5828 (Kleinmann et al. 1986). The first is located in the central zone of the cluster and would be excited by an O9 V star (Crampton et al. 1978), labelled by Russeil et al. (2007) as star number 4, and the second IRAS region, located $\sim 2$ arcmin SE from the central section, appears more extended and less populated than the central IRAS $22566+5830$.

In the Sh2-152 field of view we find five masers, all of them presented in the false-colour LIRIS image (see Fig. 2): one 6.7 GHz methanol (Szymczak et al. 2000), three water 


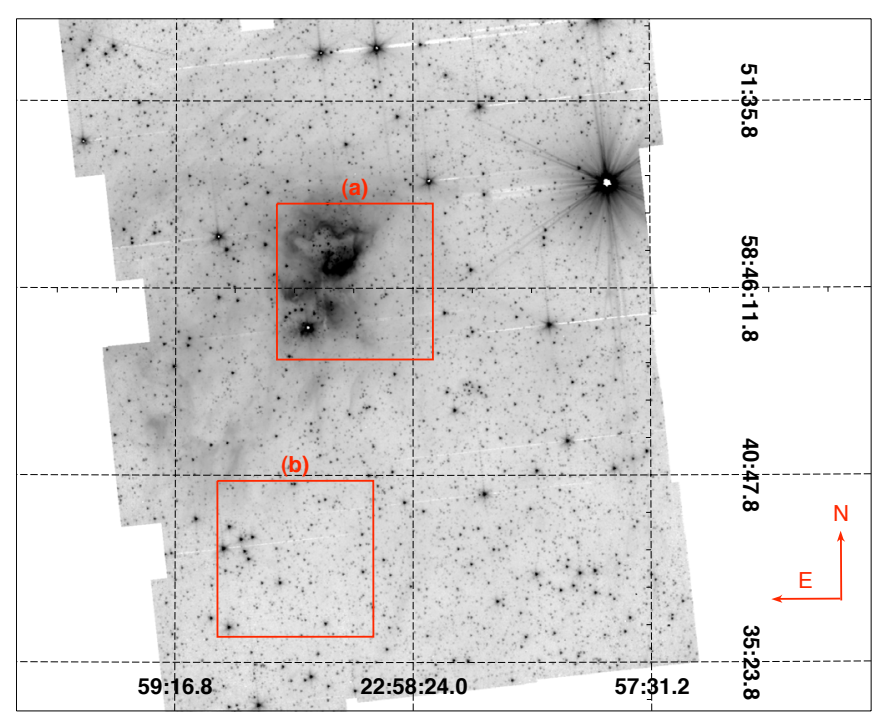

Fig. 1. Spitzer $3.6 \mu \mathrm{m}$ image. It covers the target (a) and control (b) fields for Sh2-152. Red squares are equivalent to the LIRIS field of view (4.2 arcmin length).

(Palagi et al. 1993; Harju et al. 1998), and one hydroxyl (OH) (Wouterloot \& Habing 1985). These masers are indicative of star formation and in the case of the hydroxyl one, of high-mass star formation (Zinnecker \& Yorke 2007). Its presence makes this cluster an interesting candidate as a still young and star-forming active region. Following this idea, Chen et al. (2009) used $K^{\prime}$, and $\mathrm{H}_{2}$ photometry to detect $\mathrm{H}_{2}$ emission in Sh2-152, implying that the cluster hosts ongoing star formation.

However, even if this region has been the object of nearinfrared observations its stellar content has not yet been characterized spectroscopically. Only the ionizing central star has been optically observed and spectroscopically classified (Crampton et al. 1978; Russeil et al. 2007).

Another interesting point about Sh2-152 is its distance and extension. Crampton et al. (1978), reported $3.6 \mathrm{kpc}$ as the first estimate of the cluster's distance using the spectroscopic parallax to the ionizing star. Later, in a series of studies observing $\mathrm{H}_{2} \mathrm{O}$ masers, $\mathrm{NH}_{3}$ emission lines, and both ${ }^{12} \mathrm{CO}(J=2 \rightarrow 1)$ and ${ }^{12} \mathrm{CO}(J=3 \rightarrow 2)$ transitions, Wouterloot \& Walmsley (1986) estimated the distance to Sh2-152. Assuming that Sh2152 is located in the Perseus arm, between 2 and $6 \mathrm{kpc}$ or an average distance of $3500 \mathrm{pc}$, they confirm this conjecture first with the $\mathrm{H}_{2} \mathrm{O}$ maser observation, and then, with the ammonia core (Wouterloot et al. 1988). In any case, in the third work of this series, Wouterloot et al. (1993), determined a (kinematic) distance of $5.3 \mathrm{kpc}$ through radial velocity measurements of ${ }^{12} \mathrm{CO}(J=2 \rightarrow 1)$ and ${ }^{12} \mathrm{CO}(J=3 \rightarrow 2)$ lines for IRAS $22566+5830$. In the same year, Harju et al. (1993) presented consistent results, based on observations of ammonia clumps, with the initial assumption of a distance of $3.5 \mathrm{kpc}$ for Sh2-152.

On the basis of long-slit optical spectroscopy for the central region of Sh2-152, Russeil et al. (2007) then calculated a distance of $2.39 \mathrm{kpc}$. This distance was derived from the spectral classification of the ionizing central star (the aforementioned star number 4), although we note that the position of the star is inaccurate, probably because of the spatial resolution of the associated images.

The cluster extension has been estimated considering only visual or infrared images, but from an inspection of Spitzer $3.6,4.5,5.8$, and $8.0 \mu \mathrm{m}$ images it is easy to see that the cloud around the cluster is more extended. Spectroscopy of stellar objects, covering not only the central region of Sh2-152 but the area marked with the (a) square (see Fig. 1), would allow us to determine their individual distances and the extension of the cluster.

With the mass of associated molecular clouds and highmass protostellar objects (HMPO), the mass of Sh2-152 can be indirectly estimated. Ao et al. (2004) determined the dust mass of the $\mathrm{CS},{ }^{13} \mathrm{CO}$, and $\mathrm{C}^{18} \mathrm{O}$ cores, mapping their respective lines. Assuming a distance of $3.5 \mathrm{kpc}$, from the column densities of the three observed molecular lines, they measured masses of $1.78 \times 10^{3} M_{\odot}$ (CS core), $9.20 \times 10^{3} M_{\odot}\left({ }^{13} \mathrm{CO}\right.$ core $)$, $0.29 \times 10^{4} M_{\odot}$, and $1.20 \times 10^{4} M_{\odot}$ (for two $\mathrm{C}^{18} \mathrm{O}$ cores).

From the $\mathrm{CO}$ column density and adopting a distance of $5.3 \mathrm{kpc}$ (Wouterloot et al. 1993), Guan et al. (2008) determined a mass of $3.81 \times 10^{4} M_{\odot}$ for the associated CO core, from which they observed the emergence of mass outflows. The size of the CO cloud determined by them was $4.1 \mathrm{pc}$, similar to the $4.3 \mathrm{pc}$ reported by Ao et al. (2004) to the ${ }^{13} \mathrm{CO}$ core, but larger than the other three observed cores $(1.8 \mathrm{pc}$ for the CS core and $1.7-3.0 \mathrm{pc}$ for the $\mathrm{C}^{18} \mathrm{O}$ ones). Using $850 \mu \mathrm{m}$ polarimetry, Curran et al. (2004) studied the region. For IRAS 22566+5828, they found outflows and a high-mass protostellar object (HMPO) with an estimated total mass (dust) of $3500 M_{\odot}$. In the case of IRAS $22566+5830$, they detected a magnetic field aligned north-south. In their work, they used $5.0 \mathrm{kpc}$ as the distance to the object and 100 as the gas-to-dust ratio.

As the physical parameters depend on the square of the distance, it is crucial to have a reliable determination of the distance. We therefore refine the distance and extension of Sh2152 using near-infrared photometry for the field and spectra for 13 stars. This would provide the first distance and extension estimates using more than one spectrum for candidates of the cluster stellar population. Likewise, we plan to obtain the total cluster mass by using its stellar population and luminosity function.

This paper is organized as follows. Section 2 details the observations, as well as the related data reduction procedures. The analyses of the photometry and spectroscopy (spectral classification) are described in Sect. 3. The cluster parameters are discussed in Sect. 4. Finally, Sect. 5 summarizes our conclusions.

\section{Observations}

Our study is based on broad-band infrared imaging ( $J, H$, $K_{\mathrm{S}}$ ) and medium resolution multi-object spectroscopy ( $H$ and $K)$, acquired with LIRIS, a near-infrared imager/spectrograph mounted at the Cassegrain focus of the $4.2 \mathrm{~m}$ William Herschel Telescope (Roque de Los Muchachos Observatory, La Palma).

This infrared data was complemented with optical spectroscopy for the central ionizing source of the cluster, obtained with FIES at the Nordic Optical Telescope (NOT, Roque de los Muchachos Observatory, La Palma). A summary of the observations is given in Table 1.

\subsection{Imaging}

The cluster candidate images were obtained on 2006 July 22 and the control field was observed on 2009 November 27. In both runs, seeing was between $0.58^{\prime \prime}$ and $0.69^{\prime \prime}$. Observations were carried out using LIRIS, an infrared camera equipped with a Hawaii $1024 \times 1024 \mathrm{HgCdTe}$ array detector, with a field of view of $4.2^{\prime} \times 4.2^{\prime}$ and a spatial scale of $0.25^{\prime \prime}$ pixel $^{-1}$, using $J\left(\lambda_{\mathrm{C}}=1.250 \mu \mathrm{m}, \Delta \lambda=0.160 \mu \mathrm{m}\right), H\left(\lambda_{\mathrm{C}}=1.635 \mu \mathrm{m}\right.$, 


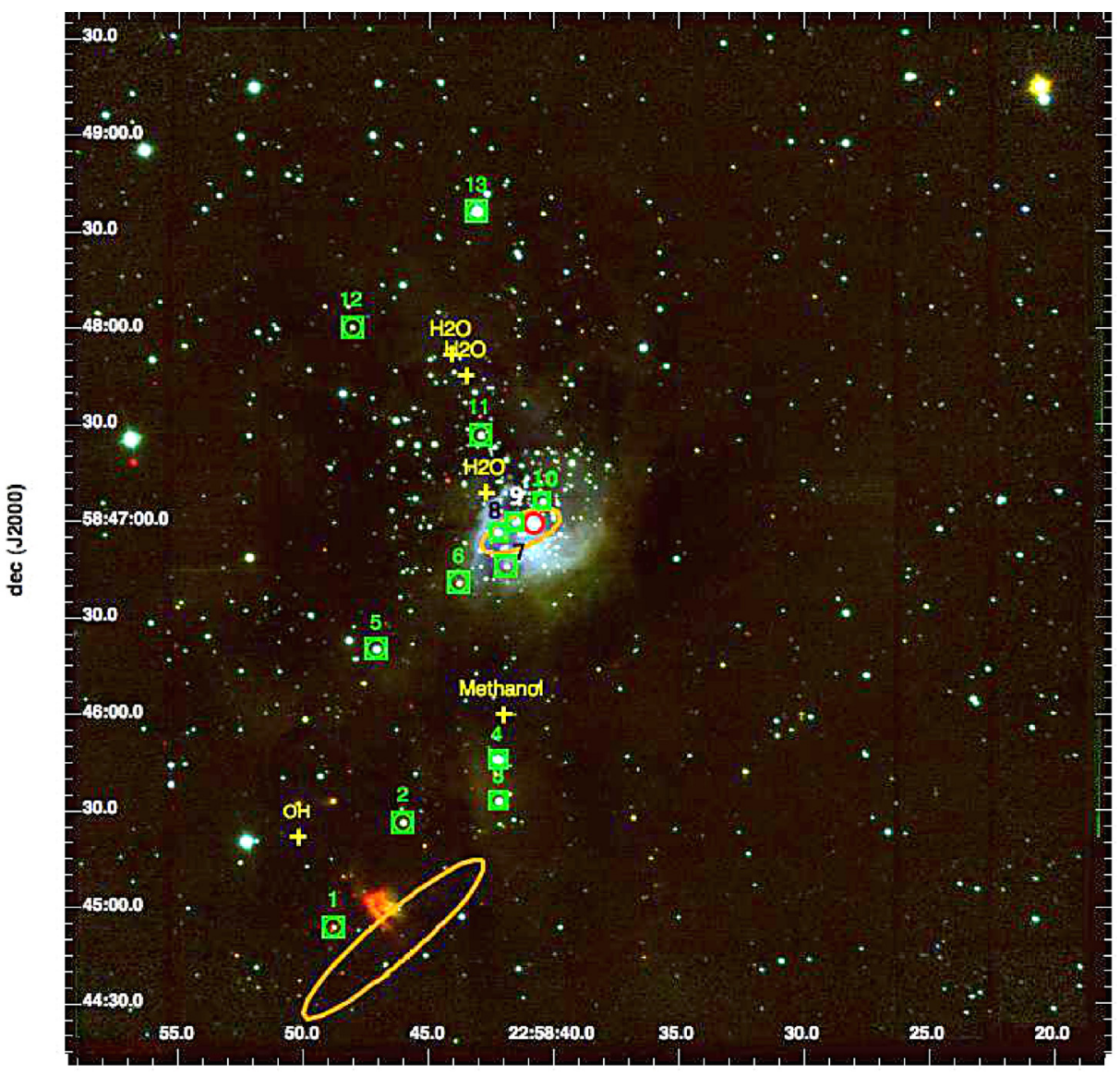

RA (J2000)
Fig. 2. LIRIS false-colour image of Sh2-152 (blue $=J$, green $=H$, red $=K_{\mathrm{S}}$ ); marked with green squares, the stars observed spectroscopically in the near-infrared. In the centre, indicated by a red circle, the central star observed spectroscopically in visual. The positions of the masers within the field are indicated by yellow crosses. The (orange) ellipses correspond to the $1-\sigma$ position uncertainties for the IRAS $22566+5830$ (central) and IRAS 22566+5828 (lower) sources. Black and white colours for central stars are used for visibility reasons.
Table 1. Summary of imaging and spectroscopic observations.

\begin{tabular}{|c|c|c|c|c|}
\hline $\begin{array}{l}\text { RA (J2000) } \\
{\left[\begin{array}{ccc}{\left[\begin{array}{ccc}\mathrm{h} & \mathrm{m}\end{array}\right]}\end{array}\right.}\end{array}$ & 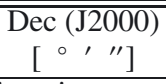 & Filter & $\begin{array}{c}\text { Exp. time } \\
{[\mathrm{s}]}\end{array}$ & $\begin{array}{c}\text { Seeing } \\
{\left[{ }^{\prime \prime}\right]}\end{array}$ \\
\hline \multicolumn{5}{|c|}{ Sh2-152 field imaging: } \\
\hline \multirow[t]{3}{*}{225837} & +584624 & $J$ & 222.30 & 0.62 \\
\hline & & $H$ & 93.12 & 0.69 \\
\hline & & $K_{\mathrm{S}}$ & 66.12 & 0.58 \\
\hline \multicolumn{5}{|c|}{ Multi-object infrared spectroscopy $(R \sim 2500)$ : } \\
\hline \multirow[t]{2}{*}{225845} & +584650 & $H$ & 2400 & 1.04 \\
\hline & & $K$ & 2400 & 0.98 \\
\hline \multicolumn{5}{|c|}{ Optical spectroscopy $(R \sim 46000)$ : } \\
\hline 225840.8 & +584658.2 & $370-730 \mathrm{~nm}$ & 1555 & 1.50 \\
\hline \multicolumn{5}{|c|}{ Control field imaging: } \\
\hline \multirow[t]{3}{*}{225850} & +583824 & $J$ & 79.34 & 0.62 \\
\hline & & $H$ & 79.34 & 0.59 \\
\hline & & $K_{\mathrm{S}}$ & 79.34 & 0.65 \\
\hline
\end{tabular}

$\Delta \lambda=0.290 \mu \mathrm{m})$, and $K_{S}\left(\lambda_{\mathrm{C}}=2.150 \mu \mathrm{m}, \Delta \lambda=0.320 \mu \mathrm{m}\right)$ filters. To improve cosmic-rays and bad-pixel rejection, and to construct the sky image for the sky subtraction, we observed in eight-point dithering mode. Data reduction (bad pixel mask, flat correction, sky subtraction, and alignment) was done with FATBOY (Eikenberry et al. 2006) and geometrical distortions were corrected with the LIRIS reduction package, LIRISDR ${ }^{1}$. The final false-colour image for Sh2-152 field is shown in Fig. 2.

The instrumental photometry was done with DAOPHOT II, ALLSTAR, and ALLFRAME (Stetson 1994), and was cleaned of non-stellar and poorly measured objects based on two

\footnotetext{
${ }^{1}$ http://www.iac.es/project/LIRIS
}

parameters. The first one is the sharpness index, which is the difference between the observed width of the object and the PSF width. Objects such as hot pixels have a negative sharpness values while a "broad" object, such as galaxies or pairs of unresolved stars, have a positive sharpness. The sharpness was complemented with the estimated standard error in the measured magnitude, $\sigma$. Using values of sharpness in the range $-0.25<$ sharp $<0.25$, and $\sigma<0.1$, we filtered $20 \%$ of the detections, all of them with $K_{\mathrm{S}}$ over 16 .

The photometric calibration (including a colour term) was done using the 2MASS catalogue (Skrutskie et al. 2006) for the three filters. We selected isolated and non-saturated stars, with photometry in 2MASS well-distributed over our whole image and the colour-magnitude diagram. For the stars with $K_{\mathrm{S}}$ brighter than 11 , we adopted the 2MASS photometry, owing to their image saturation.

The astrometric calibration was performed with SKYCAT, matching physical and equatorial coordinates for 21 sources, well-distributed in our LIRIS image. Typical values of the RMS of the fitting were smaller than 0.15 arcsec for the three bands, which is adequate for the mask design.

\subsection{Infrared spectroscopy}

The infrared spectroscopic observations were obtained on 2008 August 19 with seeing $\sim 1^{\prime \prime}$ and using the multi-object spectroscopic mode (MOS) and the HK pseudogrism, with a resolution of $\lambda / \Delta \lambda \sim 2500$. We observed two A0 V stars as telluric standards: HD 223386 for the $H$ pseudogrism observations and Hip 107555, for the $K$ one. The mask contained 13 slits of width 0.8 arcsec and length 6 arcsec and was centred at 
$\alpha_{2000}=22^{\mathrm{h}} 58^{\mathrm{m}} 45^{\mathrm{s}}$ and $\delta_{2000}=+58^{\circ} 46^{\prime} 50^{\prime \prime}$. In Fig. 2, we mark the stars observed spectroscopically in the near-IR. The selection criteria for multi-object spectrocopy is detailed in Sect. 3.1.

We observed using an ABBA strategy (a star is located in positions $\mathrm{A}$ and $\mathrm{B}$, in the slit, and then sequentially changed); with this mode, we are able to remove the sky from the spectra by simply subtracting spectra at position A from spectra at position $\mathrm{B}$, and vice versa. Flat-fielding, tracing, sky subtraction, coaddition, and extraction were done using LIRISDR, a package developed specifically for LIRIS data that uses the information from the mask design files. Thanks to the combination of the individual spectra (eight for each band), we discarded cosmic rays and hot pixels that could mimic spectral lines. For wavelength calibration, argon and xenon lamps were observed, using both lamps (continuum subtracted) to calibrate the $K$-band spectra and the argon lamp for $H$-band calibration.

Telluric subtraction was done using XTELLCOR (Vacca et al. 2003), an IDL program that, applying a high-resolution synthetic model of an A0 V star (the spectral type of our standard) over the observed telluric standards, produces the calibration spectrum with the telluric lines. This spectrum was then used to correct our science spectra, with the IRAF $^{2}$ task TELLURIC.

\subsection{Optical spectroscopy}

The optical spectra for the central ionizing source of Sh152-2 (star number 4 from Russeil et al. 2007) was obtained on 2009 November 12, using the FIES cross-disperser high-resolution echelle spectrograph, at the $2.5 \mathrm{~m}$ NOT. We used FIES in medium resolution mode $(R=46000)$, with a fibre size of $1.3^{\prime \prime}$ and the spectral range of 370 to $730 \mathrm{~nm}$ covered in a single fixed setting. The exposure time for the individual spectra was $25.83 \mathrm{~min}$, and the seeing during the observations was $1.5 \mathrm{arcsec}$.

\section{Results}

Previous near-infrared studies of Sh2-152 were based on the 2MASS photometry, thus their results were limited to magnitude $K_{\mathrm{S}} \sim 13$. With our new observations, we obtained near-infrared photometry with limiting magnitude $K_{\mathrm{S}} \sim 20$ for the cluster and control field. These data were used to select the OB-type star candidates from the cluster main-sequence for the spectroscopic follow-up.

\subsection{Colour-magnitude diagram}

The cluster candidate and control field colour-magnitude diagrams are shown in Fig. 3. In these diagrams we see how the cluster's main sequence is scattered following the reddening vector due to the differential reddening over the field. This scatter also affects part of the local dwarf sequence, which is the principal feature in the control field CMD. Because of the differential extinction over the candidate field, cluster stars have redder $\left(J-K_{\mathrm{S}}\right)$ colours and dimmer $K_{\mathrm{S}}$ magnitudes.

The selection of OB-type candidates considers this effect by selecting stars with $\left(J-K_{\mathrm{S}}\right)$ in the range $0.5-4.5$ and $K_{\mathrm{S}}<13$.

\footnotetext{
2 IRAF is distributed by the National Optical Astronomy Observatories, which are operated by the Association of Universities for Research in Astronomy, Inc., under cooperative agreement with the National Science Foundation.
}

This first requirement would select stars embedded in the cluster's natal cloud or their own particular natal cloud, while the second requirement comes from the instrumental limitation of spectra of high signal-to-noise $(S N R)$. It is necessary to keep in mind that telluric variations impose an upper limit on the infrared exposure times.

For the candidate selection, we also took into account the positions of these stars over the field of view. First of all, the cluster's boundaries do not appear to be clearly defined in the LIRIS near-IR images. Including stars that are not only in the central region of the image would help us to derive information about the membership of stars separated from the central part of Sh2-152. Secondly, the number and positions of slits in the mask is limited by spectral overlap in the dispersion axis and the spectral features that we wish to observe.

From the target and control field CMDs, it is also possible to estimate roughly the cluster candidate distance. Observing both CMDs, we can identify the local dwarf sequence, formed by dwarf stars situated in the solar neighbourhood, as a vertical strip between $\left(J-K_{\mathrm{S}}\right)=0.4$ and $\left(J-K_{\mathrm{S}}\right)=1.0$. This strip looks sharper for the control field CMD in the whole $K_{\mathrm{S}}$ magnitude range but, in the target CMD, this sequence spreads for $K_{\mathrm{S}}>15$. The effect can be understood as the extra reddening caused by Sh2-152 over the disc stars located behind the cluster candidate. Hence, any star from the local sequence brighter than the magnitude where this sequence widens would be located between the cluster candidate and us. By locating this point, we can make a first estimation of the distance to Sh2-152.

A method of obtaining this estimate is presented in Marín-Franch et al. (2009). Considering 11.2 \pm 0.75 Gyr as the characteristic age for stars in the solar neighbourhood (Binney et al. 2000), in their CMDs they plot isochrones with this age at different distances and thus under different extinctions. They then locate the point where target and control field local sequences start to differentiate into this set of isochrones. The closest isochrone to this point indicates a rough distance estimate for the cluster.

After including three isochrones in our CMDs for 3.0, 4.0, and $5.0 \mathrm{kpc}$, we can see that differences appear between the 3.0 and $4.0 \mathrm{kpc}$ isochrones (Fig. 3). Therefore, a first estimate of the distance of Sh2-152, completely independent of the spectrophotometric distance estimate, would be less than $4.0 \mathrm{kpc}$.

\subsection{Completeness tests}

To precisely remove the disc stellar population from the cluster's luminosity function, and quantify the instrumental sensitivity to stellar magnitudes and colours, we carried out a completeness test, as described in Hidalgo et al. (2008) and Aparicio \& Gallart (1995).

Completeness tests consist of adding artificial stars to the images and then, repeating the photometry using the same parameters and procedures as for target and control field photometry. For our tests, we included 480200 artificial stars over 200 images (for $J$ and $K_{\mathrm{S}}$ filters) with $K_{\mathrm{S}}$ instrumental magnitudes between 12 and 22 and colour $\left(J-K_{\mathrm{S}}\right)$ between 0 and 6; with these limits, we homogeneously covered the whole range of the CMDs. To avoid the creation of extra crowding, the stars were added following a network, within the full range of coordinates into the image, and using a star-to-star distance of two times the PSF radius plus one. In this array, star positions are fixed from image to image, but $J$ and $K_{\mathrm{S}}$ magnitudes vary.

Finally, for each artificial star of magnitude $K_{\mathrm{S}, 1}$ and colour $\left(J-K_{\mathrm{S}}\right)_{1}$, we defined a section of $K_{\mathrm{S}, 1} \pm 0.4$ and $\left(J-K_{\mathrm{S}}\right)_{1} \pm 0.4$, 


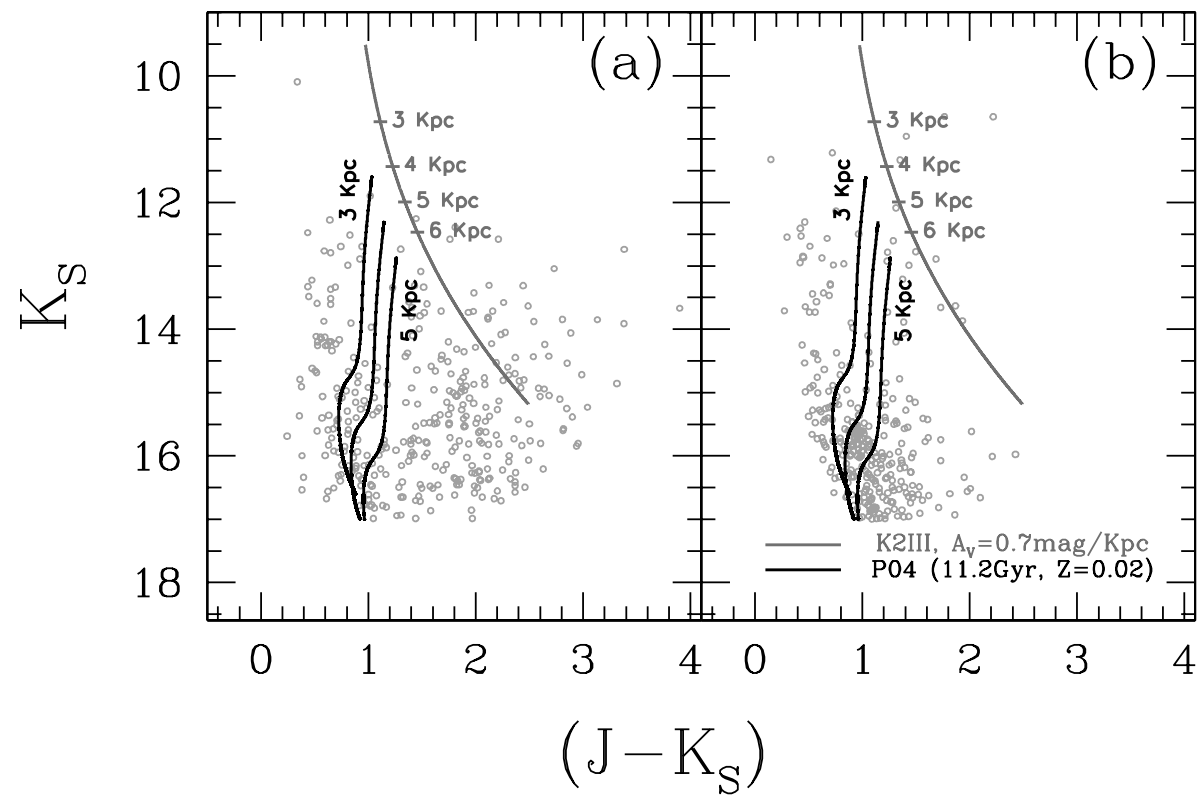

Fig. 3. Calibrated colour-magnitude diagrams for Sh2-152 a) and the control field b). The black lines represent isochrones of solar metallicity and $11.2 \mathrm{Gyr}$, located at three distances $(3.0,4.0$ and $5.0 \mathrm{kpc})$ and the grey curve, the position of a K2 III star at several distances. The isochrone was generated using the Pietrinferni et al. (2004) stellar evolutionary library, the Castelli \& Kurucz (2003) bolometric correction library and the Rieke et al. (1989) extinction law with $R=3.09$ (Rieke \& Lebofsky 1985). For the K2 III sequence, $M_{V}$ was obtained from Cox (2000) and IR intrinsic colours from Ducati et al. (2001). where we counted the number of recovered and injected stars. The ratio of these numbers is the completeness index assigned to that star. The completeness test reproduces the detection limit for the CMD and gives a completeness of more than 0.90 for over $97 \%$ of the CMD. The value of the completeness factor according to the position in the CMD is shown in Fig. 4. It can be seen in the top diagram that for $K_{\mathrm{S}}$ fainter than 16.5 , the completeness drops to 0.8 , meaning that $80 \%$ of the stars would be detected at that magnitude.

\subsection{Near-infrared spectral classification}

We based our near-IR spectral classifications for the OB stellar types on those of Hanson et al. (1996) for the $K$-band and Hanson et al. (1998) for the $H$-band, and for the later spectral types on those of Meyer et al. (1998) and Wallace \& Hinkle (1997). The characteristic lines for each spectral type were complemented with a visual comparison between our spectra and other spectral catalogues, with the $H$ - and $K$-bands at similar resolutions (Ivanov et al. 2004; Ranade et al. 2004; Ranada et al. 2007; Hanson et al. 2005). Table 2 contains the coordinates, near-infrared magnitudes, and spectral types of the spectroscopically observed stars. The positions in the CMD and CC diagrams for the observed stars are presented in Figs. 5 and 6.

Spectral classification in the $K$-band can be problematic. The main feature in this band for late B to early F-type stars is Brackett $\gamma$ at $2.166 \mu \mathrm{m}$, but this line is adjusted in the A0 V modelling when constructing the telluric-line correction spectra. Any over- or under-correction could therefore add noise to this particular line. Complementing the analysis with the $H$-band spectral information overcomes this problem.

Another consideration stems from the nebular differential emission that dominates the central zone of the cluster. This affects the stellar spectra in two ways. First, the emission can contaminate the spectra, making it hard to distinguish the nature of the lines, whether stellar or nebular. This is noticeable in star \#8, whose wide Brackett-series emission lines seem to be contaminated with a narrow absorption line. This absorption line is an artefact resulting from the nebular contribution included in the slit and reversed through the sky subtraction.
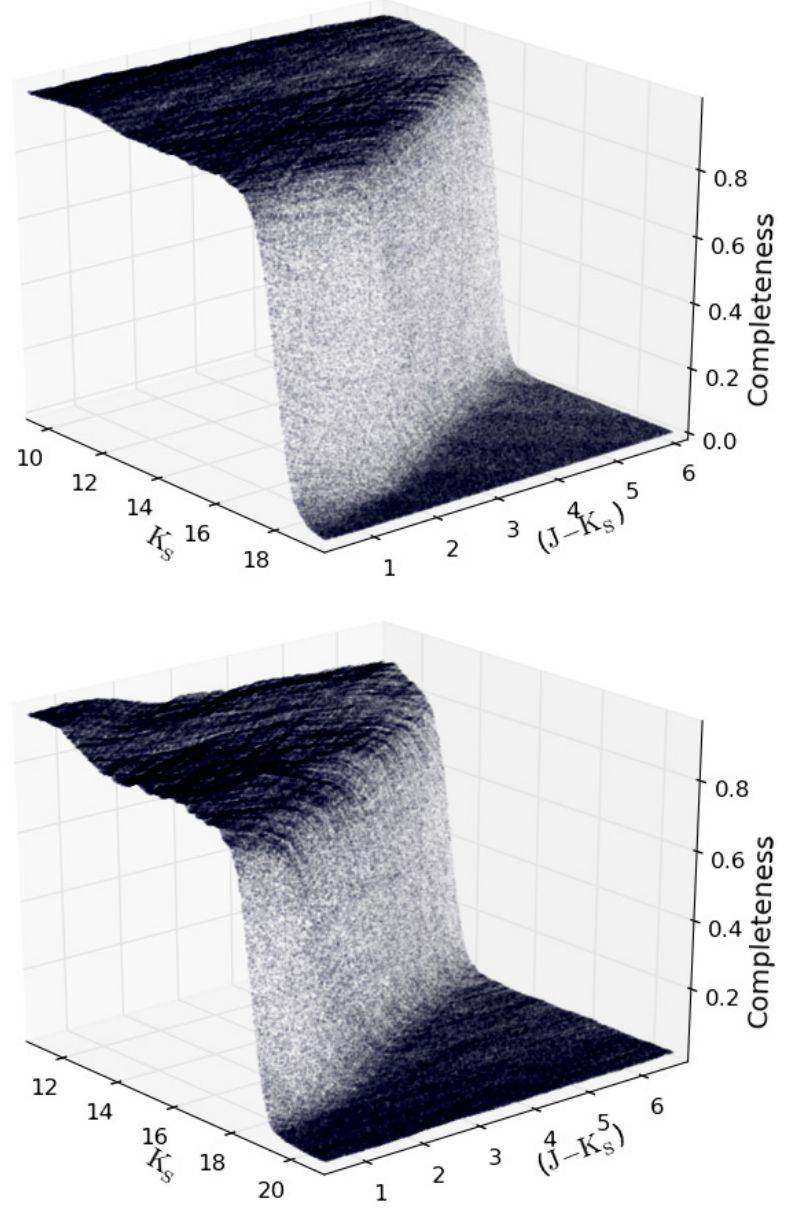

Fig. 4. Surface formed by the points in colour-magnitude-completeness space is shown. The top figure corresponds to the Sh2-152 field and the bottom figure, to the control field. Completeness ranges from 0 to 1 .

The second problem is that, owing to the inhomogeneous nebular distribution, for the same slit we can have sky with an added nebular contribution relative to the local sky or even no nebular contribution to the star position. This causes an artificial 


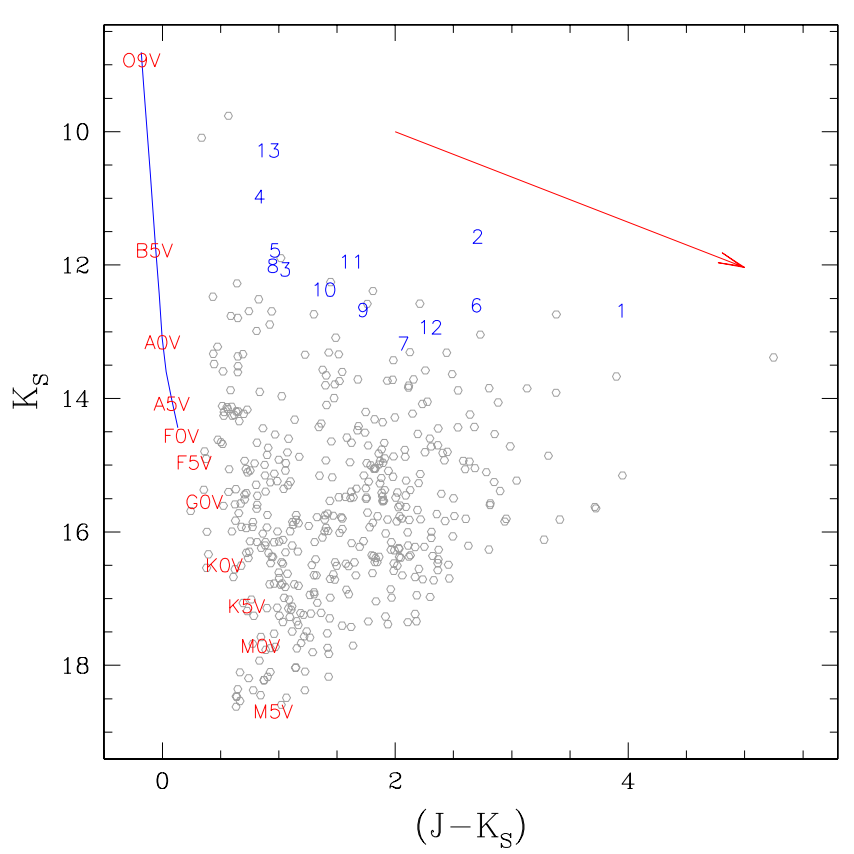

Fig. 5. Calibrated colour-magnitude diagram for Sh2-152. The red segmented arrow shows $A_{V}=20 \mathrm{mag}\left(A_{K s}=2.04\right)$ and the main sequence is located at the distance determined in this study $(3.21 \mathrm{kpc})$. The continuous blue line in the upper part of the main sequence corresponds to the best fit made to project the CMD stars to the main sequence, following the reddening vector. The position of the spectroscopically observed stars is marked with blue numbers.

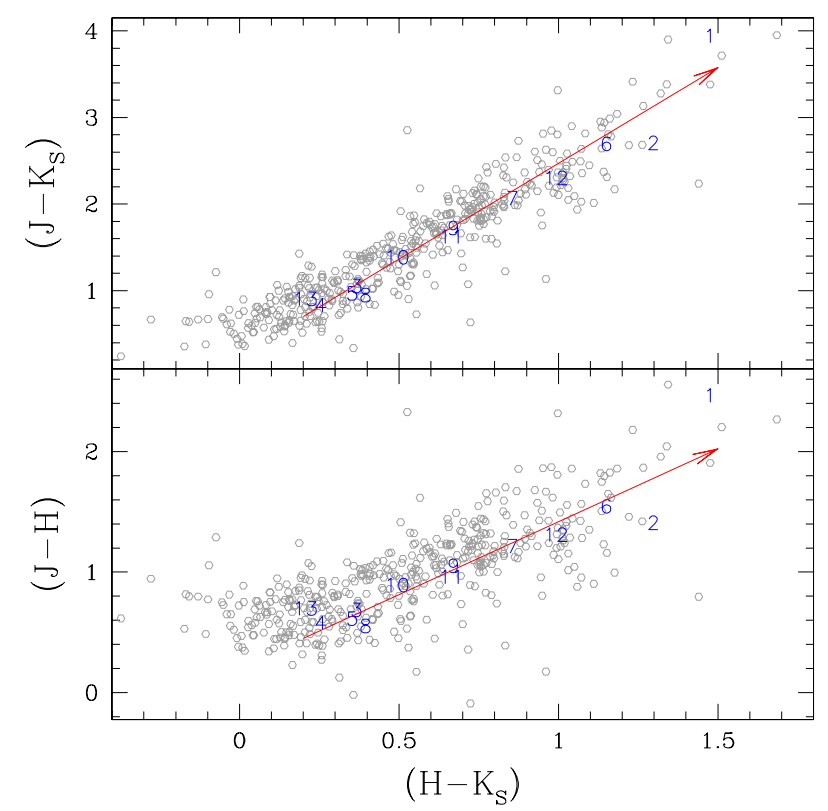

Fig. 6. Calibrated colour-colour diagrams for Sh2-152. The blue numbers corresponds to the spectroscopically observed stars. Red arrows in both diagrams show the reddening vector.

subtraction of nebular lines or, in some cases, an artificial emission line. This effect is easy to detect through a visual inspection of the individual A-B or B-A spectra, detecting lines that pass from absorption to emission as we change from an A-B to a B-A image. Examples of spectra with this behaviour are numbers 7 and 10 , both located in the central region of the cluster, where inhomogeneous nebular emission is dominant. The final near-IR spectra are shown in Fig. 7.

Star \#1 is located in a very interesting sector of the field. Its surroundings look to be clear in the $J$-band but for $K_{\mathrm{S}}$ a bright arc and a nebulosity arises. This arc even rivals the central zone of Sh2-152 in brightness (Spitzer ch-4, $8.0 \mu \mathrm{m}$ ). Because of this nebulosity, star \#1 is the most reddened star in our set but we cannot determine whether it is the ionizing star of the IRAS $22566+5828$. The star was classified as B1 V, based on the shape and depth of its $\operatorname{Br} \gamma$ line and the presence of helium $(2.11 \mu \mathrm{m})$. The $H$-band is quite noisy, and the HeI $1.70 \mu \mathrm{m}$ is contaminated by nebular emission. Owing to this noise, produced by its lower position in the detector causing the lost of half of the flux, the stellar type could easily be two subtypes earlier or later.

Spectrum \#2 is characterized by its strong emission Brackett lines. This object is located below the main-sequence line in the colour-colour diagrams (Fig. 6). Its near-IR colour excess, which is characteristic of YSOs, and the dominating emission lines over the whole spectra lead us to classify it as a YSO.

Stars \#3 and \#4 have similar features and their spectral types vary in the range B2-3 V according to their $H$-band spectra. Star \#3 spectra is noisier than star \#4, and this explain the weaker Brackett series. However for both spectra the series are present until $\operatorname{Br} 15(1.57 \mu \mathrm{m})$, confirming the similar spectral types of both stars. We also detect for both stellar spectra the HeI $1.70 \mu \mathrm{m}$ line. The $K$-band spectrum does not clearly display the HeI $2.11 \mu \mathrm{m}$ line, but the $\mathrm{Br} \gamma$ line is again consistent with a B2-3 V spectral classification.

For star \#5, the spectral type is difficult to define. The spectrum clearly shows the lines $\mathrm{Br} 12, \mathrm{Br} \gamma$, and $\mathrm{HeI}$ at $1.70 \mu \mathrm{m}$ but a weak Brackett series. This is characteristic of late $\mathrm{O}$ - to early B-dwarfs. Comparing with spectra from the Hanson catalogues, we find that the spectral type of this object corresponds to B2 V.

In the case of stars \#6 $\left(H=13.75\right.$ and $\left.K_{\mathrm{S}}=12.66\right)$ and $\# 7\left(H=14.03\right.$ and $\left.K_{\mathrm{S}}=13.24\right)$, it was impossible to derive a precise spectral type owing to the presence of some emission lines, the lack of other strong spectral features, and the low $S N R$ of our data for these dim objects.

In the centre of the cluster, nebular emission is an important contributor to the spectra and, in some objects, appears to be inhomogeneous. Star \#8, practically in the middle of the field, has been resolved thanks to our LIRIS image resolution. The nebular emission is overcorrected because of the inhomogeneities in the sky emission around the object. The hydrogen lines exhibit a wide emission component mixed with shallower absorption. This might be due to the overlapping of the nebular (wide) contribution with stellar (narrow) lines. We also detect HeI at 1.7 and $2.06 \mu \mathrm{m}$ for this object. The position of the star \#8 in the colour-colour diagram, below the main-sequence line, indicates that it is a YSO-like star \#2. Because it is less reddened, it might have cleared its surroundings.

A similar effect could occur for star \#9; its spectra looks featureless in absorption, probably because its lines are filled by the nebular emission lines. The classification of the spectrum, dominated by Brackett and HeI emission lines, was impossible.

Star \#10 has a well-defined Brackett series, with no indication of either helium or carbon lines. A comparison with star HR5685 from Ranade's library leads us to classify this object as B5 V.

Stars \#11 and \#12 are late-type stars, even if the CO bands do not clearly appear in these spectra. A close similarity can be found between star \#11 and Ranade's HR5019 (spectral type G6.5 V) and between star \#12 and Meyer's HR4496 (spectral type G8 V). Both appear to be stars in the Galactic disc. 


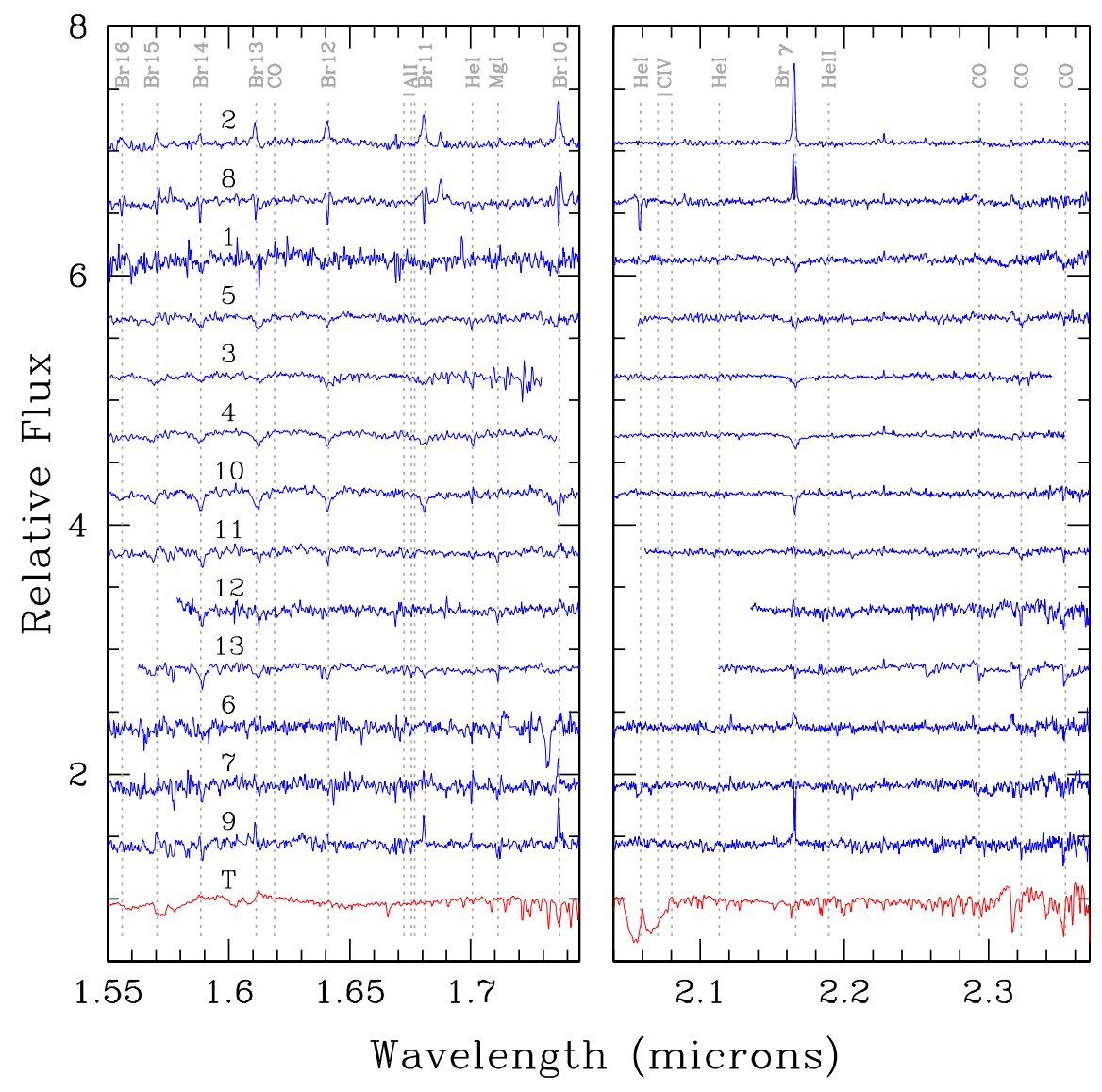

Fig. 7. Individual spectra for the observed Sh2152 stars. The spectra are arranged, from top to bottom, into YSOs, early types, late types, and those without spectral classification. The final red spectrum corresponds to the telluric correction.

Table 2. Spectroscopically observed stars.

\begin{tabular}{ccccccc}
\hline \hline ID & $\begin{array}{c}\text { RA (J2000) } \\
{\left[\mathrm{h} \mathrm{m}^{\mathrm{s}}\right]}\end{array}$ & $\begin{array}{c}\text { Dec (J2000) } \\
{\left[{ }^{\circ}{ }^{\prime}{ }^{\prime}\right]}\end{array}$ & $\begin{array}{c}J \\
{[\mathrm{mag}]}\end{array}$ & $\begin{array}{c}H \\
{[\mathrm{mag}]}\end{array}$ & $\begin{array}{c}K_{\mathrm{S}} \\
{[\mathrm{mag}]}\end{array}$ & Spectral type \\
\hline 1 & 225848.774 & +584453.72 & 16.598 & 14.158 & 12.745 & B1 V \\
2 & 225846.007 & +584526.23 & 14.258 & 12.870 & 11.633 & YSO \\
3 & 225842.189 & +584532.88 & 13.131 & 12.434 & 12.127 & B2-3 V \\
4 & 225842.221 & +584545.68 & 11.821 & 11.229 & 11.034 & B2-3 V \\
5 & 225847.081 & +584620.00 & 12.758 & 12.134 & 11.840 & B2 V \\
6 & 225843.785 & +584640.59 & 15.284 & 13.754 & 12.665 & $\ldots$ \\
7 & 225841.859 & +584645.99 & 15.242 & 14.035 & 13.240 & $\ldots$ \\
8 & 225842.215 & +584656.47 & 12.967 & 12.407 & 12.071 & YSO \\
9 & 225841.541 & +584659.56 & 14.395 & 13.343 & 12.732 & $\ldots$ \\
10 & 225840.422 & +584706.06 & 13.762 & 12.863 & 12.430 & B5 V \\
11 & 225842.920 & +584726.67 & 13.572 & 12.614 & 12.010 & G6.5 V \\
12 & 225848.062 & +584800.22 & 15.221 & 13.923 & 12.992 & G8 V \\
13 & 225843.076 & +584836.34 & 11.203 & 10.491 & 10.344 & G8-9 III \\
\hline
\end{tabular}

Finally, star \#13 has a remarkable position in the colourmagnitude diagram, being the brightest star in our selection. However, the weak Brackett and the strongest $\mathrm{CO}$ heads in our candidates indicate a late-type for this star. The spectra looks similar to those of HR5888 (Ivanov et al. 2004) and HR7328 (Meyer et al. 1998). We classify star \#13 as G8-G9 III.

\subsection{Spectral classification (optical)}

Our final optical spectrum for the central star of Sh2-152, is shown in Fig. 8. This star was previously classified as O9 V by Crampton et al. (1978) and O8.5 V by Russeil et al. (2007).

Our spectral classification is based on the study of Walborn \& Fitzpatrick (1990). On first inspection, the spectral type for the central star of Sh2-152 appears to be O9-B0 V star, because of its ratio of HeI $4471 \AA$ to HeII $4542 \AA$. However, there are a few spectral features in the optical spectra that deserve some review.

First of all, the HeII $4686 \AA$ line is similar in depth to the HeI $4471 \AA$ line and deeper than the HeI $4388 \AA$ one. The ratio HeII $4686 \AA$ HeI-4471 A looks slightly larger than for stars between O9 V and B0 V. An HeII $4686 \AA$ spectral line stronger than that centred on HeI $4471 \AA$ A would indicate a Vz type (Walborn 2009) and might indicate that there is ongoing massive-star formation in the cluster.

Secondly, the CNO complex at 4630-4650 A looks slightly fainter than expected for those spectral types. Although this 


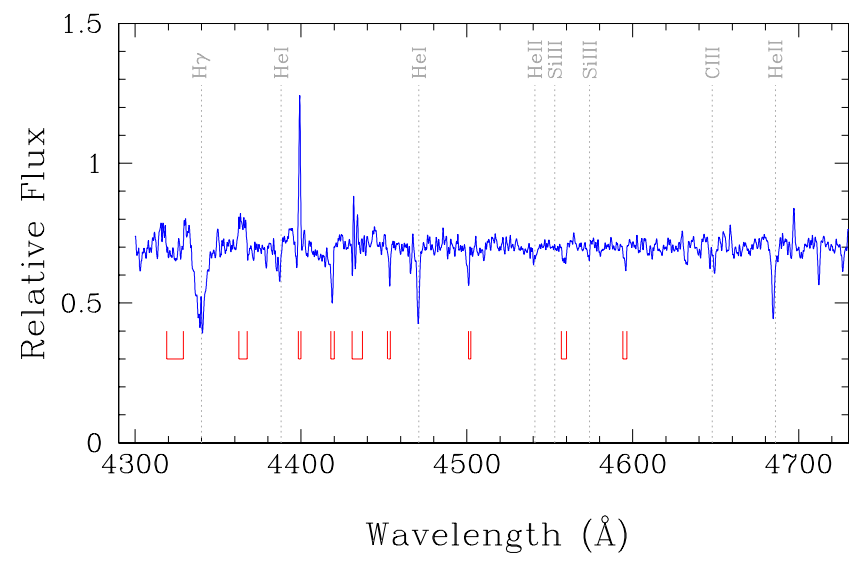

Fig. 8. Optical spectrum for the central star of Sh2-152 (Russeil's star number 4). Vertical segmented lines (grey) mark the spectral lines used in the spectral classification. Overlapping zones of the spectral orders are marked with red vertical lines; the low $S N R$ causes a poor combination of the spectral orders, which could mimic a spectral line. Spectra resolution was decreased to $R=8000$, to help us to discern the lines.

might be caused by the continuum rectification, this would not seem to be sufficient to justify the difference.

The third interesting feature is the absence of a SiIII (4553-4574 $\AA$ ) detection. These lines appear for spectral types later than $09.5 \mathrm{~V}$, hence this detection indicates a spectral type of $\mathrm{O} 9 \mathrm{~V}$ or earlier. However, for types earlier than the aforementioned limit, the depths of the HeII $4542 \AA$ and HeII $4686 \AA$ lines do not fit.

Therefore, the star was assigned a spectral type of O9-B0 V, with spectral features that are not fully consistent with any of the subtypes. While the $S N R$ of the spectrum is relatively modest (the $S N R$ per pixel is 20, but at $R=46000$, which allows us to degrade our spectrum without loss of information), we cannot exclude the presence of a binary system, a composite spectrum, or a slightly peculiar spectrum. The possibility of a $\mathrm{Vz}$ type would also enhance the peculiarity of this star, but data of higher $S N R$ is mandatory to confirm this point.

\section{Discussion}

\subsection{Distance and size determinations}

Using the estimated spectral types, we derived the individual distances to cluster stars. Assuming the absolute visual magnitudes of Cox (2000), the intrinsic infrared colours of Tokunaga (2000), and the Cardelli et al. (1989) extinction law with $R=3.10$, the extinction for the $K_{\mathrm{S}}$ band can be expressed as

$A_{K_{\mathrm{S}}}=\frac{E_{J-K_{\mathrm{S}}}}{1.474}=\frac{E_{H-K_{\mathrm{S}}}}{0.667}$.

Across the field of view, we can find several situations of extinction ranging from 0.62 to $2.70 \mathrm{mag}$ for $A_{K_{\mathrm{S}}}$, or $A_{V}$ between 5.44 and $23.68 \mathrm{mag}$. In Table 3 , we present the values of $A_{K_{\mathrm{S}}}$ and the individual distances of the early-type stars used in the distance estimation.

We excluded Russeil's star number 4 (central star of Sh2152) because it appears saturated in our images, and 2MASS images of this object are unable to resolve the central zone of the cluster. The 2MASS magnitudes for the central star would be contaminated and we prefer to exclude this object from the distance estimation.

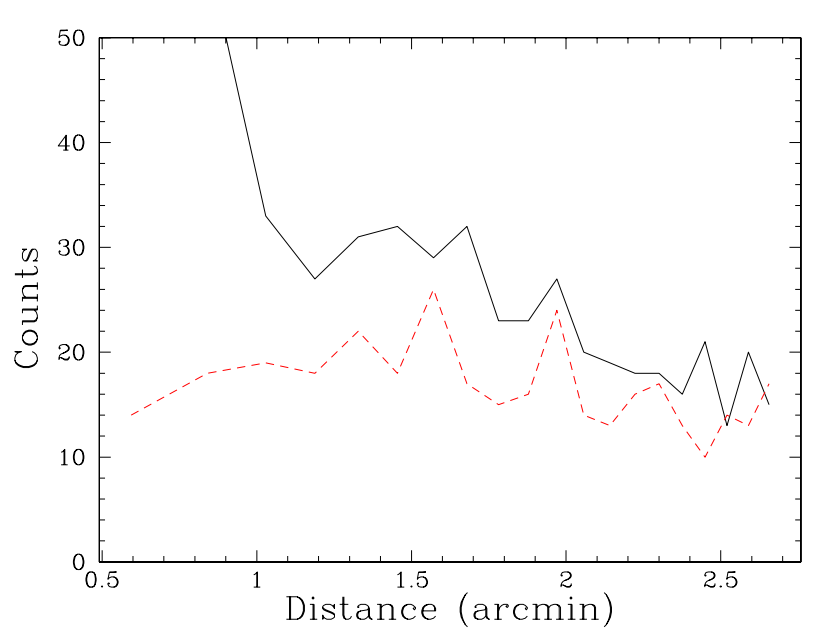

Fig. 9. Radial distribution of stars for Sh2-152 (solid black curve) and the control field (red dashed curve).

Table 3. Reddening and distances of early-type stars.

\begin{tabular}{lccc}
\hline \hline Spectral type & ID & $\begin{array}{c}A_{K_{\mathrm{S}}} \\
{[\mathrm{mag}]}\end{array}$ & $\begin{array}{c}\text { Distance } \\
{[\mathrm{kpc}]}\end{array}$ \\
\hline B1 V & 1 & 2.70 & 3.17 \\
B2 V & 5 & 0.70 & 3.76 \\
B2-3 V & 3 & 0.76 & 3.61 \\
B2-3 V & 4 & 0.62 & 2.70 \\
B5 V & 10 & 0.95 & 2.83 \\
\hline
\end{tabular}

With the five early-type stars, we obtained an average distance to the cluster of $3.21 \pm 0.21 \mathrm{kpc}$ from the individual calculations. This value is larger than that given by Russeil et al. (2007) $-D=2.39 \mathrm{kpc}-$ and slightly smaller than the distance of $3.5 \mathrm{kpc}$ estimated by Crampton et al. (1978). Both estimates were obtained from spectral observations of the central ionizing star of the cluster (our O9-B0 V star). In our case, the estimate was derived from individual distance estimates that are self-consistent and cover a wider area of the cluster.

From the Spitzer image of the field and our observation of a nearby control field using LIRIS, we learned that the angular size of the cluster is comparable to the LIRIS field of view $\left(4.2^{\prime} \times 4.2^{\prime}\right)$. The cluster was previously considered as just the surroundings of Sh2-152, underestimating the size of the object, but from our CMD, we realize that stars near the edge of our image are part of the cluster. Hence, considering at least a cluster size of 4.2' (the LIRIS field of view) and the average distance of $3.21 \mathrm{kpc}$, the diameter of the cluster would be $\gtrsim 4.0 \mathrm{pc}$.

A test that helps us to clarify the extension of the cluster is to count the number of stars for different concentric rings covering the whole field of view. Taking as centre the coordinates of IRAS $22566+5830$, we counted the sources in concentric rings of 25 arcsec in thickness. In Fig. 9, we can see that there is an evident change in the slope of the counts in the central section of the image (around 250 pixels, i.e. 60 arcsec), but even as the number of stars detected continues to decrease it remains larger than the number of stars detected for the control field. In addition, the existence of stars with the same spectrophotometric distances as objects within the central area of the cluster allows us to confirm that the size of the cluster is at least comparable to our field of view, and that the subtraction of stellar counts using the same field is unreliable. 


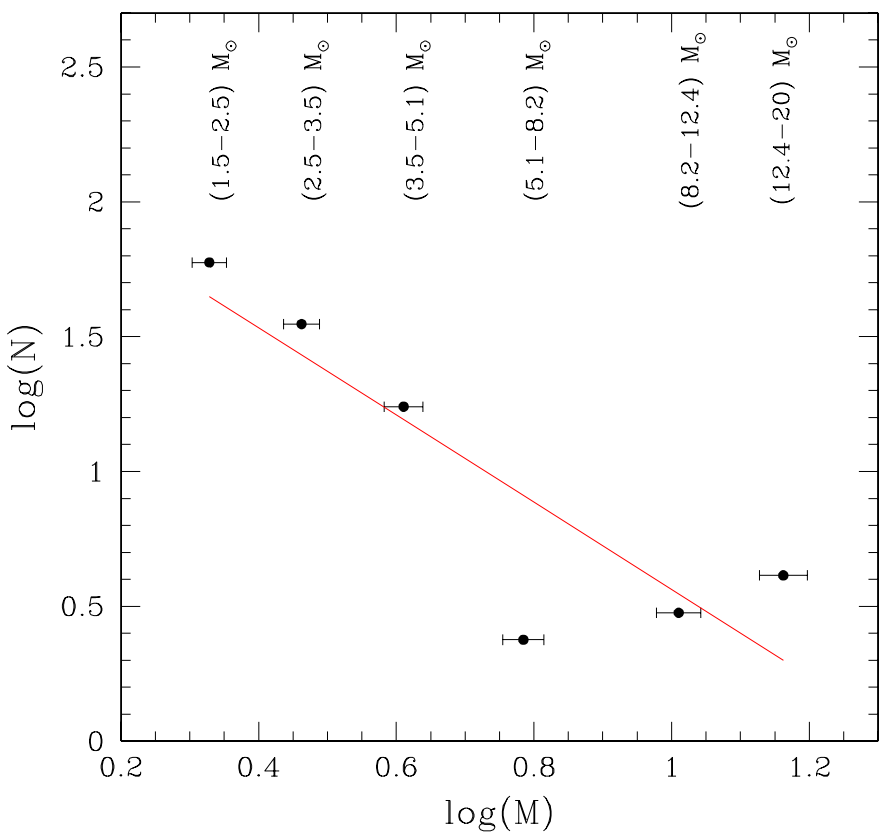

Fig. 10. Mass function for Sh2-152. The points indicate the central position of the mass range indicated above, equivalent to $K_{\mathrm{S}}$ magnitude bins of 0.8 . The continuous red line shows the best linear fit to the data.

\subsection{Cluster mass and age estimations}

To derive the cluster mass, we integrated the stellar mass function of Sh2-152, previously corrected for the field stellar contribution, after accounting for the effect of completeness on the star counts.

The first step in obtaining the mass function is to project every star following the reddening vector, to the dwarf star sequence defined by the magnitudes and colours of Cox (2000) and located at our estimated distance. This sequence can be expressed analytically by a second degree function or a set of first degree functions. We found that fitting the dwarf sequence using two lines, one from $\mathrm{O} 9 \mathrm{~V}$ to $\mathrm{A} 0 \mathrm{~V}$ and the second from $\mathrm{A} 0 \mathrm{~V}$ to F0 V (blue solid lines in Fig. 5) was more effective than fitting a second-degree function. The cut in F0 V arises from our detection limit; at later spectral types (fainter magnitudes), the histograms start to decline towards lower masses.

Once the stars in the Sh2-152 and the control field CMDs were projected following the reddening vector to the line-fitted main sequence, we produced the histograms including the specific weight for each star, according to its completeness factor determined by its position in the CMD $\left(J-K_{\mathrm{S}}, K_{\mathrm{S}}\right)$. The correction for the incompleteness consists simply of multiplying the number of stars by the inverse of the completeness factor.

The histograms bins were 0.8 in $K_{\mathrm{S}}$, equivalent to twice the size of the completeness bins. To convert a $K_{\mathrm{S}}$ magnitude to a stellar mass, we used the values given by Cox (2000). For inbetween magnitudes catalogued values, we interpolated between the two closest values.

After subtracting both histograms (i.e. subtracting the control field histograms from the Sh2-152 field), we derived the cluster mass function (Fig. 10). Deriving the best-fit linear relation $(\Gamma=-1.62)$ in the $\log (N)-\log (M)$ plane and then integrating from 0.20 to $20 M_{\odot}$, we obtained a total mass for Sh2-152 of $(2.45 \pm 0.79) \times 10^{3} M_{\odot}$.

To compare this result with the literature, we had to carefully consider the nature of both quantities. Our value comes from evaluating the stellar content of Sh2-152, in sharp contrast to studies such as Ao et al. (2004), Guan et al. (2008), and Curran et al. (2004), who derived the virial mass, i.e. the stellar mass necessary to ensure that the molecular core is gravitationally bound, from molecular radial velocities.

Guan et al. (2008) calculated the mass (dust) for the associated CO core. Their value for the total mass (dust) is $3.81 \times$ $10^{4} M_{\odot}$, an order of magnitude higher than our result. If we use our distance estimate instead of the value used by Guan et al. (2008) $(5.3 \mathrm{kpc})$, the total mass (dust) for the CO core drops to $1.22 \times 10^{4} M_{\odot}$ and its virial mass is also reduced by a factor of 0.32 , down to $3.04 \times 10^{3} M_{\odot}$.

The study of Ao et al. (2004) assumes a distance that is more similar to our estimate. With $d=3.5 \mathrm{kpc}$, they obtained four total mass (dust) estimates: $1.78 \times 10^{3} M_{\odot}$ for the CS core, $9.20 \times$ $10^{3} M_{\odot}$ for ${ }^{13} \mathrm{CO}$ core, and $(0.29-1.20) \times 10^{4} M_{\odot}$ for the two $\mathrm{C}^{18} \mathrm{O}$ cores. With the exception of the virial mass derived from the ${ }^{13} \mathrm{CO}$ estimate $\left(4.90 \times 10^{3} M_{\odot}\right)$, their associated virial masses are lower than those obtained by Guan et al.: $1.78 \times 10^{3} M_{\odot}$ (for $\mathrm{CS}$ core) and $(0.67-1.40) \times 10^{3} M_{\odot}$ (for the $\mathrm{C}^{18} \mathrm{O}$ cores).

With the stellar mass and dust mass estimates, we can evaluate the cluster's star formation efficiency (SFE). The star formation efficiency is the ratio of the cluster's stellar mass to the gas-stellar mass. To derive the total gas mass associated with the dust total mass, we used the gas-to-dust ratio. According to Curran et al. (2004), this ratio varies from 45:1 to 100:1, so we prefer to use both values in the estimate.

Using the dust mass measured by Curran et al. (2004), projected to our estimated distance instead of the $5.0 \mathrm{kpc}$ used by them, we obtained the associated gas mass, and estimated the SFE to be between $1.7 \%$ and $3.7 \%$. This value is similar to that reported by Hunter et al. (1990) (i.e. 6\%), assuming a distance of $4.0 \mathrm{kpc}$ and estimating the stellar total mass with a normalization of the IMF to the upper stellar range, which in their case is 18-25 $M_{\odot}$.

We can neither precisely determine the age of the cluster nor confirm the idea of sequential stellar evolution (Chen et al. 2009), but we can constrain the cluster's age based on the physical characteristics of its stellar population. First of all, our earliest main-sequence star is of type O9 V. Since it has not evolved away from the main sequence, the cluster lifetime is shorter than the time spent by this star on the main sequence. Assuming that $18 M_{\odot}$ is the mass of an $\mathrm{O} 9 \mathrm{~V}$ star (Martins et al. 2005) and that the cluster population was formed at the same time, we can only limit the cluster's age to be younger than 9.4 Myr, using the stellar models for a $20 M_{\odot}$ solar metallicity star of Schaller et al. (1992). This limit is obviously conservative and cannot be considered an age estimate.

\section{3. lonizing sources}

We used our spectral type classification together with data collected from the literature to estimate whether the total ionizing photons emitted by the stars can explain the radio observations of Sh2-152. To determine the number of ionizing photons $\left(\log Q_{0}\right)$ for O-type stars, we used Martins et al. (2005). For B0 V stars, we used the values given by Simón-Díaz \& Stasińska (2008) and for the B0.5-B1 V spectral types we took the radii from the binary CW Cep (Bozkurt \& Değirmenci 2007), the spectral type- $T_{\text {eff }}$ relation from Lefever et al. (2010), and the $T_{\text {eff }}-\log q_{0}$ from the B-star grid from Lanz \& Hubeny (2007).

To derive the number of Lyman continuum photons from the radio density flux, $N_{\mathrm{LyC}}$, we used Eq. (A.5) of Hunt et al. (2004), assuming an electron temperature of $T=10^{4} \mathrm{~K}$ and 
$n\left(\mathrm{He}^{+}\right) \ll n\left(\mathrm{H}^{+}\right)$. Since this formula is for radiation-bounded ionized HII regions, it is not guaranteed that it is applicable to IRAS $22566+5828$, although an estimation may be illustrative.

We divide the ionizing stars into two groups: the first contains stars in the central region $\left(r \sim 30^{\prime \prime}\right)$, including IRAS 22566+5830, star \#10 and Russeil's star number 4. The earliest star in this region is Russeil's number 4 (O9-B0 V, this work), with a total number of ionizing photons between $\log Q_{0}=48.06$ and 47.29. The number of Lyman continuum photons derived from the radio observations for IRAS $22566+5830$ is $\log N_{\mathrm{LyC}}=$ 48.02 (Condon et al. 1998), which is most consistent with an O9 V spectral classification for the central star of Sh2-152.

The second region, formed by stars \#1, \#3, \#4, and \#5, is located close to IRAS $22566+5828$ source and its earliest star is star \#1. Within our sample, star \#1 would therefore be the main ionizing source associated with IRAS $22566+5828$. However, the value of $\log Q_{0}=45.66$ for this star is significantly lower than for the Lyman continuum photons, $\log N_{\text {LyC }}=48.35$, derived from the radio observations (Harju et al. 1998) indicating that the star \#1 does not produce enough photons to explain the radio observations. The $K_{\mathrm{S}}$ image shows a deeply embedded star in the centre of the red nebulosity close to of IRAS $22566+5828$, which appears to be the $\mathrm{O} 7.5-8 \mathrm{~V}$ star required as the single ionizing source of this region. Future $K$ spectroscopy for this star would help us to confirm this result.

\section{Conclusions}

In the framework of our MASGOMAS project, we have completed a spectrophotometric study of the stellar content of the compact HII region Sh2-152 using LIRIS at the WHT and the FIES cross-disperser echelle spectrograph at the NOT.

Our new near-infrared data have allowed us to resolve the central part of the cluster, to select OB candidates for $H$ and $K$ spectroscopy, and estimate their individual distances. The mean of the individual distances to five B-type stars, $3.21 \pm 0.21 \mathrm{kpc}$, places the cluster slightly closer than the estimates obtained by Crampton et al. (1978); Wouterloot \& Walmsley (1986); Wouterloot et al. (1988), and Harju et al. (1993) but definitely further away than estimated by Russeil et al. (2007). In any case, our estimated distance is in closer agreement with the distance estimates based on the central ionizing star (star number 4 in Russeil et al. 2007) than with those derived from ${ }^{12} \mathrm{CO}$ radial velocities.

We also obtained the cluster mass function, corrected for the Galactic disc stellar contribution (using the observed control field) and for completeness effects (through artificial star injection and photometry). Integrating this function, we calculated a lower limit to the cluster total mass. The value of $(2.45 \pm 0.79) \times 10^{3} M_{\odot}$ agrees with the mass values from the literature, all of them being virial masses necessary to reproduce the observed molecular velocities (for both $\mathrm{CO}$ and $\mathrm{CS}$ cores).

The central O9-B0 V star remaining on the main sequence allows us to limit the cluster's age to be younger than 9.4 Myr. The optical spectra for this central star displays several interesting features. First, the strenght of its helium lines (HeII $4686 \AA$ similar to that of HeI $4471 \AA$ and stronger than HeI $4388 \AA$ ) might be indicative of a $\mathrm{V} z$ spectral type, although the relation between the HeII $4686 \AA$ line and HeII $4542 \AA$ line appears to exclude this. Secondly, the spectra detect a fainter CNO complex at 4630-4650 $\AA$ for the derived spectral type. And third, we have found the absence of SiIII (4553 $\AA$ and $4574 \AA$ A). Either a binary system, a composite spectrum, or a slightly peculiar spectrum are possible explanations of the last two features. Future observations of this object with higher $S N R$ will help us to shed light on the real nature of the central, most massive star of Sh2-152.

For two regions of Sh2-152, we have compared the number of ionizing photons from the classified OB-stars with the number of Lyman continuum photons derived from radio observations. For the central region containing both IRAS $22566+5830$ and the O9-B0 V central star (Russeil's star number 4), both of these numbers are consistent with there being a single ionizing source.

For the second region containing IRAS $22566+5828$, the number of Lyman continuum photons cannot be explained by the earliest star in our sample, and the derived value of $\log N_{\mathrm{LyC}}$ would imply the existence of an 07.5-8 V star. This source could be a highly embedded star, without spectral classification, at the centre of the bright red arc close to star \#1. We expect future spectral observations of this star to help us verify this finding.

Acknowledgements. S.R.A. was supported by the MAEC-AECID scholarship. Part of this work was supported by the Science and Technology Ministry of the Kingdom of Spain (grants AYA2008-06166-C03-01), and the Fundación Agencia Aragonesa para la Investigación y Desarrollo (ARAID). S.R.A., A.H., A.M.-F., E.P., F.N., J.A.A.P., and S.S. are members of the Consolider-Ingenio 2010 Program (CSD2006-00070).

The William Herschel Telescope is operated on the island of La Palma by the Isaac Newton Group in the Spanish Observatorio del Roque de los Muchachos of the Instituto de Astrofísica de Canarias. This publication makes use of data products from the Two Micron All Sky Survey, which is a joint project of the University of Massachusetts and the Infrared Processing and Analysis Center/California Institute of Technology, funded by the National Aeronautics and Space Administration and the National Science Foundation.

\section{References}

Alexander, M. J., Kobulnicky, H. A., Clemens, D. P., et al. 2009, AJ, 137, 4824 Ao, Y., Yang, J., \& Sunada, K. 2004, AJ, 128, 1716 Aparicio, A., \& Gallart, C. 1995, AJ, 110, 2105

Benjamin, R. A., Churchwell, E., Babler, B. L., et al. 2003, PASP, 115, 953

Bica, E., Dutra, C. M., \& Barbuy, B. 2003a, A\&A, 397, 177

Bica, E., Dutra, C. M., Soares, J., \& Barbuy, B. 2003b, A\&A, 404, 223

Binney, J., Dehnen, W., \& Bertelli, G. 2000, MNRAS, 318, 658

Bozkurt, Z., \& Değirmenci, Ö. L. 2007, MNRAS, 379, 370

Cardelli, J. A., Clayton, G. C., \& Mathis, J. S. 1989, ApJ, 345, 245

Castelli, F., \& Kurucz, R. L. 2003, in Modelling of Stellar Atmospheres, ed. N. Piskunov, W. W. Weiss, \& D. F. Gray (San Francisco: ASP), IAU Symp., 210, Poster A20

Chen, Y., Yao, Y., Yang, J., Zeng, Q., \& Sato, S. 2009, ApJ, 693, 430

Clark, J. S., Negueruela, I., Crowther, P. A., \& Goodwin, S. P. 2005, A\&A, 434, 949

Clark, J. S., Negueruela, I., Davies, B., et al. 2009, A\&A, 498, 109

Condon, J. J., Cotton, W. D., Greisen, E. W., et al. 1998, AJ, 115, 1693

Cox, A. N. 2000, Allen's Astrophysical Quantities, 4th edition (New York: Springer)

Crampton, D., Georgelin, Y. M., \& Georgelin, Y. P. 1978, A\&A, 66, 1

Curran, R. L., Chrysostomou, A., Collett, J. L., Jenness, T., \& Aitken, D. K. 2004, A\&A, 421, 195

Davies, B., Figer, D. F., Kudritzki, R.-P., et al. 2007, ApJ, 671, 781

Davies, B., Figer, D. F., Law, C. J., et al. 2008, ApJ, 676, 1016

Ducati, J. R., Bevilacqua, C. M., Rembold, S. B., \& Ribeiro, D. 2001, ApJ, 558, 309

Dutra, C. M., \& Bica, E. 2001, A\&A, 376, 434

Dutra, C. M., Bica, E., Soares, J., \& Barbuy, B. 2003, A\&A, 400, 533

Eikenberry, S., Elston, R., Raines, S. N., et al. 2006, in Ground-based and Airborne Instrumentation for Astronomy, ed. I. S. McLean, \& M. Ian (Washington: SPIE), Proc. SPIE, 6269, 626917

Epchtein, N., de Batz, B., Capoani, L., et al. 1997, The Messenger, 87, 27

Figer, D. F. 2008, in Massive Stars as Cosmic Engines, ed. F. Bresolin, P. A. Crowther, \& J. Puls (Cambridge: Cambridge Univ. Press), IAU Symp., 250, 247

Figer, D. F., Kim, S. S., Morris, M., et al. 1999, ApJ, 525, 750

Figer, D. F., MacKenty, J. W., Robberto, M., et al. 2006, ApJ, 643, 1166

Froebrich, D., Scholz, A., \& Raftery, C. L. 2007, MNRAS, 374, 399 
Guan, X, Wu, Y, \& Ju, B. 2008, MNRAS, 391, 869

Hanson, M. M., \& Popescu, B. 2008, in Massive Stars as Cosmic Engines, ed. F. Bresolin, P. A. Crowther, \& J. Puls (Cambridge: Cambridge Univ. Press), IAU Symp., 250, 307

Hanson, M. M., Conti, P. S., \& Rieke, M. J. 1996, ApJS, 107, 281

Hanson, M. M., Rieke, G. H., \& Luhman, K. L. 1998, AJ, 116, 1915

Hanson, M. M., Kudritzki, R., Kenworthy, M. A., Puls, J., \& Tokunaga, A. T. 2005, ApJS, 161, 154

Harju, J., Walmsley, C. M., \& Wouterloot, J. G. A. 1993, A\&AS, 98, 51

Harju, J., Lehtinen, K., Booth, R. S., \& Zinchenko, I. 1998, A\&AS, 132, 211

Hidalgo, S. L., Aparicio, A., \& Gallart, C. 2008, AJ, 136, 2332

Hunt, L. K., Dyer, K. K., Thuan, T. X., \& Ulvestad, J. S. 2004, ApJ, 606, 853

Hunter, D. A., Thronson, Jr., H. A., \& Wilton, C. 1990, AJ, 100, 1915

Ivanov, V. D., Rieke, M. J., Engelbracht, C. W., et al. 2004, ApJS, 151, 387

Kleinmann, S. G., Cutri, R. M., Young, E. T., Low, F. J., \& Gillett, F. C. 1986, IRAS Serendipitous Survey Catalog (Tucson: IPAC)

Knödlseder, J. 2000, A\&A, 360, 539

Lanz, T., \& Hubeny, I. 2007, ApJS, 169, 83

Lawrence, A., Warren, S. J., Almaini, O., et al. 2007, MNRAS, 379, 1599

Lefever, K., Puls, J., Morel, T., et al. 2010, A\&A, 515, A74

Marín-Franch, A., Herrero, A., Lenorzer, A., et al. 2009, A\&A, 502, 559

Martins, F., Schaerer, D., \& Hillier, D. J. 2005, A\&A, 436, 1049

Mercer, E. P., Clemens, D. P., Meade, M. R., et al. 2005, ApJ, 635, 560

Meyer, M. R., Edwards, S., Hinkle, K. H., \& Strom, S. E. 1998, ApJ, 508, 397

Negueruela, I., González-Fernández, C., Marco, A., Clark, J. S., \& MartínezNúñez, S. 2010, A\&A, 513, A74

Palagi, F., Cesaroni, R., Comoretto, G., Felli, M., \& Natale, V. 1993, A\&AS, 101,153
Pietrinferni, A., Cassisi, S., Salaris, M., \& Castelli, F. 2004, ApJ, 612, 168

Ranada, A. C., Singh, H. P., Gupta, R., \& Ashok, N. M. 2007, Bulletin of the Astronomical Society of India, 35, 87

Ranade, A., Gupta, R., Ashok, N. M., \& Singh, H. P. 2004, Bulletin of the Astronomical Society of India, 32, 311

Rieke, G. H., \& Lebofsky, M. J. 1985, ApJ, 288, 618

Rieke, G. H., Rieke, M. J., \& Paul, A. E. 1989, ApJ, 336, 752

Russeil, D., Adami, C., \& Georgelin, Y. M. 2007, A\&A, 470, 161

Schaller, G., Schaerer, D., Meynet, G., \& Maeder, A. 1992, A\&AS, 96, 269

Simón-Díaz, S., \& Stasińska, G. 2008, MNRAS, 389, 1009

Skrutskie, M. F., Cutri, R. M., Stiening, R., et al. 2006, AJ, 131, 1163

Stetson, P. B. 1994, PASP, 106, 250

Szymczak, M., Hrynek, G., \& Kus, A. J. 2000, A\&AS, 143, 269

Tokunaga, A. T. 2000, in Allen's Astrophysical Quantities, ed. A. N. Cox, 4th edition (New York: Springer), 143

Vacca, W. D., Cushing, M. C., \& Rayner, J. T. 2003, PASP, 115, 389

Walborn, N. R. 2009, in Massive Stars: From Pop III and GRBs to the Milky Way, ed. M. Livio, \& E. Villaver (Cambridge: Cambridge University Press), 167

Walborn, N. R., \& Fitzpatrick, E. L. 1990, PASP, 102, 379

Wallace, L., \& Hinkle, K. 1997, ApJS, 111, 445

Wouterloot, J. G. A., \& Habing, H. J. 1985, A\&A, 151, 297

Wouterloot, J. G. A., \& Walmsley, C. M. 1986, A\&A, 168, 237

Wouterloot, J. G. A., Walmsley, C. M., \& Henkel, C. 1988, A\&A, 203, 367

Wouterloot, J. G. A., Brand, J., \& Fiegle, K. 1993, A\&AS, 98, 589

Zinnecker, H., \& Yorke, H. W. 2007, ARA\&A, 45, 481 